Orthopäde 2016 · 45:1039-1044

DOI 10.1007/s00132-016-3293-2

Online publiziert: 8. Juli 2016

(c) Der/die Autor(en) 2016. Dieser Artikel ist

eine Open-Access-Publikation.

CrossMark

\begin{abstract}
Wirbelsäulenchirurgische Eingriffe erfordern ein hohes Maß an Konzentration. Gleichzeitig können starke körperliche und mentale Belastungen auftreten. Bisher gibt es keine systematischen Untersuchungen oder Daten zu Kreislaufparametern von Wirbelsäulenchirurgen in der Rolle des Operateurs oder des Assistenten unter Berücksichtigung unterschiedlicher physischer und psychischer Belastungen während wirbelsäulenchirurgischer Eingriffe.
\end{abstract}

\section{Einleitung}

Komplexe Operationen können weit über $10 \mathrm{~h}$ andauern. Oft sind Pausen zu kurz oder kommen erst gar nicht in Betracht, da diese aufgrund des Operationsablaufs nicht möglich sind. In der Regel steht der Chirurg während der gesamten Zeit des Eingriffs am Operationstisch. Er ist dementsprechend einer hohen physischen Anstrengung ausgesetzt.

Diese Anstrengung steigt durch das Tragen von OP-Bekleidung, Mundschutzmaske, Kopflampe und - wie es oft bei orthopädischen Operationen notwendig ist - von röntgenstrahlendichter Bleischürze mit hohem Eigengewicht zusätzlich. Hieraus resultiert zudem eine eingeschränkte Bewegungsfreiheit. Während die OP-Bekleidung mit Hose und Kasack einen eher geringen Einfluss hat, fällt die Bleischürze mit knapp $5,5 \mathrm{~kg}$ deutlicher ins Gewicht. Die Operationsleuchte, die dem Arzt ein optimales Sichtfeld auf das Operationsgebiet gewähren soll, wird trotz des klimatisierten

\author{
J. Kremer ${ }^{1,2} \cdot$ M. Reinhold ${ }^{1}$ \\ ${ }^{1}$ Univ.-Klinik für Orthopädie, Medizinische Universität Innsbruck, Innsbruck, Österreich \\ ${ }^{2}$ Elz, Deutschland
}

\title{
Intraoperativer Stress bei Wirbelsäuleneingriffen
}

\section{Operateur vs. Assistent}

Operationssaals aufgrund ihrer enormen Leuchtstärke von bis zu 160.000 Lux [16] in unmittelbarer Nähe zum Operateur zu einer beachtlichen Wärmequelle. Ein besonderes Augenmerk sollte auch auf das Tragen der Mundschutzmaske gelegt werden, da diese $\mathrm{zu}$ einer merklichen thermischen Zusatzbelastung führen kann [4, 8, 10, 11].

Zum Aufgabengebiet eines Wirbelsäulenchirurgen gehört ein vielfältiges Repertoire an Operationen. Neben minimalinvasiven Operationen mit einem Operationsmikroskop, bei denen ein enormes $\mathrm{Maß}$ an Feingefühl während der Arbeit an sehr kleinen anatomischen Strukturen gefragt ist, gibt es auch Eingriffe, bei denen grobmotorisches, handwerkliches Geschick und Kraft im Vordergrund stehen. Insbesondere das Ausharren in einer unphysiologischen, gebückten oder schrägen Zwangshaltung des Oberkörpers, Nackens oder Kopfes bedeutet eine Herausforderung für die statische Muskelarbeit.

Zusätzlich entsteht psychischer Stress, wenn der Eingriff ein hohes Maß an Konzentration über einen mehrstündigen Zeitraum erfordert. Die Korrekturen einer ausgeprägten Wirbelsäulendeformität oder Tumorerkrankung nehmen nicht selten einen ganzen Arbeitstag in Anspruch. Das mit solchen Eingriffen verbundene hohe perioperative Risiko für Komplikationen, z. B. für eine Querschnittslähmung oder Blutung bei Gefäßverletzungen, erhöht den psychischen Druck und bedeutet ein hohes Maß an Verantwortung gegenüber dem Patienten und seinen Angehörigen.
In der gegenwärtigen Literatur existieren nur wenige Untersuchungen, welche die intraoperativen Kreislaufparameter, den Dehydratationsgrad des Chirurgen sowie deren Auswirkungen im Hinblick auf psychischen und physischen Stress systematisch untersucht und analysiert haben. Im Vergleich mit medizinischen Berufen sind die Luftfahrt und andere Berufsfelder mit hoher Verantwortung, bei denen durch menschliche Fehler, Ermüdung und Konzentrationsschwäche fatale Konsequenzen drohen, wesentlich besser erforscht. Durch Forschungsarbeiten im Luftfahrtsektor konnten typische Fehlerquellen identifiziert und deren Auftreten durch das Multi-Crew-Concept, einem Schulungsprogramm zur Verbesserung von Kommunikation und Koordination unter Kopilot und Pilot, auf ein Mindestmaß reduziert werden. So gehört die professionelle Luftfahrt heute zu den sichersten Reise- und Transportmodalitäten [9]. Hier kommt es bei einer Million Flügen zu weniger als 0,15 fatalen Unfällen, was zu einem großen Teil auf ein gut ausgebautes Risikomanagement und das Bewusstsein der Belastungen von Pilot und Kopilot zurückzuführen ist [9].

Betrachtet man hingegen den Krankenhausreport der AOK [17] von 2014, in dem laut Schätzungen rund 19.000 Menschen im Jahr ihr Leben aufgrund von Behandlungsfehlern verlieren, wird deutlich, dass ein dringender Handlungsbedarf besteht, um Komplikationen durch Verbesserungen am Arbeitsplatz und Risikobewusstsein während chirurgischer Eingriffe zu minimieren.

Die Ergebnisse der vorliegenden Arbeit sollen zu einem besseren Verständnis 
Tab. 1 Kreislaufparameter, OP-Dauer und Kalorienverbrauch von Operateur und Assistent

\begin{tabular}{|lll}
\hline & Operateur & Assistent \\
\hline Anzahl der Operationen & 73 & 28 \\
\hline Durchschnittliche Herzfrequenz (spm) fehlende $\mathrm{n}=4$ & $98^{\mathrm{a}}$ & 76,5 \\
\hline Maximale Herzfrequenz $(\mathrm{spm})$ fehlende $\mathrm{n}=7$ & $123,6^{\mathrm{a}}$ & 99,3 \\
\hline Durchschnittliche Dauer (in $\mathrm{h})$ & $3: 05$ & $2: 50$ \\
\hline Durchschnittlicher Energieverbrauch $(\mathrm{kcal})$ & $740^{\mathrm{a}}$ & 367,7 \\
\hline${ }^{\mathrm{a}}$ statistisch signifikante Unterschiede $(p<0,05)$ & & \\
\hline
\end{tabular}

der Arbeitsplatzbelastungen im Kontext von physischem und psychischem Stress während wirbelsäulenchirurgischer Eingriffe beitragen.

\section{Methode}

Die Studie umfasste eine Analyse relevanter Kreislaufparameter eines gesunden, 40-jährigen Wirbelsäulenchirurgen mit 12 Jahren Praxiserfahrung (BMI 25) während 101 Operationen unterschiedlichen Schwierigkeitsgrads im Zeitraum vom Dezember 2011 bis März 2013 an der Universitätsklinik für Orthopädie der medizinischen Universität Innsbruck. Die Operationen wurden entweder "aktiv“ als erster Operateur durchgeführt oder „passiv“ in der Rolle des Assistenten begleitet.

\section{Ablauf}

Das jeweils aktuelle Körpergewicht wurde mithilfe einer Personenwaage (Fa. Korona, Modell Dolores) unmittelbar vor und direkt nach der Operation gemessen. Dabei wurde standardisiert vorgegangen. Die Messungen erfolgten jeweils entkleidet nach dem Toilettengang mit entleerter Blase und vor dem Anlegen der Operationsbekleidung. Während der Operationen waren keine Trinkpausen oder Toilettengänge vorgesehen. Die Messung des Körpergewichts nach Beendigung der Operation wurde nach Ablegen der Operationskleidung durchgeführt.

Die Messung der Herzfrequenz durch einen digitalen Trainingscomputer $\left(\right.$ Polar $^{\circledR}$ RS300X) startete beim Betreten des Waschraums und endete beim Verlassen des Operationssaals. Um den Einfluss der Umgebungstemperatur während des Eingriffs festzustellen, wurde die Raumtemperatur zu Beginn und am Ende der
Operation mit einem Kühlraumthermometer gemessen und dokumentiert.

Die primäre Datenerfassung erfolgte mit einem Erfassungsbogen. Die Daten wurden zur digitalen Weiterverarbeitung in eine Excel-Tabelle übertragen und mit SPSS ausgewertet.

\section{Messparameter}

Gemessen wurden folgende Parameter:

- durchschnittliche Herzfrequenz in Schlägen pro Minute,

- maximale Herzfrequenz in Schlägen pro Minute,

- Gesamtkalorienverbrauch in Kilokalorien,

- Raumtemperatur in Grad Celsius,

- Gewicht des Probanden in Kilogramm.

Der Schwierigkeitsgrad der Operation wurde auf einer Skala von eins bis drei bewertet, wobei eins als „leicht“ (operationstechnisch einfache, häufig durchgeführte Routineeingriffe, z. B. mikroskopisch assistierte Bandscheibenoperation, dorsale Dekompression einer Segmentetage) und drei als „schwer“ (operationstechnisch anspruchsvolle, seltene, mit höherem Komplikationsrisiko behaftete Eingriffe, z. B. langstreckige Korrekturspondylodesen, Revisionseingriffe mit Narbenbildung).

\section{Dokumentation}

Für jede durchgeführte Operation wurde ein separater Erfassungsbogen ausgefüllt, der die zuvor beschriebenen Messparameter enthält. Zusätzlich wurden in dem Erfassungsbogen Komplikationen erfasst, so denn diese auftraten. Das Tragen einer Strahlenschutzweste wurde protokolliert und die Funktion des Probanden als Operateur (aktiv) oder
Assistent (passiv) notiert. Die Art des Eingriffs, die OP-Dauer, der Beginn und das Ende (Uhrzeit in hh:mm) der Messungen wurden ebenfalls festgehalten.

Die mit dem Trainingscomputer erfassten Daten wurden digital mit dem vom Hersteller dafür vorgesehenen Polar Flowlink ${ }^{\circledR}$, einem computergestützten System mit passwortgeschütztem Onlineaccount (www.polarpersonaltrainer. com), ausgelesen. Alle Datenblätter wurden im Studienbuch gesammelt und archiviert.

\section{Ergebnisse}

Die statistische Auswertung umfasste 101 Operationen. Dabei wurden physische und psychische Aspekte der intraoperativen Belastung berücksichtigt.

\section{Physischer Stress}

Die Auswirkungen auf die körperliche Belastung wurde anhand der gemessenen Herzfrequenz ( $n=97$, fehlende $n=4)$ in der Rolle des „aktiven“ Operateurs und „passiven“ Assistenten verglichen. In der Funktion als Assistent betrug sie im Mittel $77 \pm 6 \mathrm{spm}$ (Schläge pro Minute). Als aktiver Chirurg betrug sie $98 \pm 10 \mathrm{spm}$. Die durchschnittliche Herzfrequenz war somit bei den aktiven Operationen statistisch signifikant höher als bei den passiven ( $p \leq 0,05$, Mann-Whitney-U-Test). Bei der maximalen Herzfrequenz gab es ebenfalls signifikante Unterschiede hinsichtlich der aktiven und passiven Rolle des Operateurs während der Eingriffe ( $p \leq 0,05$, Mann-Whitney-U-Test). Der Mittelwert betrug $99 \pm 11$ spm bei assistierten Operationen und $124 \pm 15$ spm bei aktiven Operationen ( $\bullet$ Tab. 1).

Die Gewichtsabnahme durch den Verlust von Körperflüssigkeit wurde mithilfe einer wiederholten Messung des Körpergewichts vor und unmittelbar nach der Operation bestimmt. Die Flüssigkeitsverluste von insgesamt 99 Messungen reichten von $0 \mathrm{~kg}$ bis $2,3 \mathrm{~kg}$. Dies entspricht $0 \%$ bis $2,68 \%$ des Körpergewichts. Der Mittelwert lag bei $0,82 \pm 0,61 \mathrm{~kg}(0,97 \%$ des Körpergewichtes $\pm 0,71 \%$ ).

Es bestand eine hohe Korrelation ( $\mathrm{r}=$ 0,64) zwischen der Dauer der Operation und dem Gewichtsverlust. Der errechne- 
Orthopäde 2016 · 45:1039-1044 DOI 10.1007/s00132-016-3293-2

(c) Der/die Autor(en) 2016. Dieser Artikel ist eine Open-Access-Publikation.

J. Kremer · M. Reinhold

Intraoperativer Stress bei Wirbelsäuleneingriffen. Operateur vs. Assistent

\section{Zusammenfassung}

Einleitung. Lange wirbelsäulenchirurgische Eingriffe stellen eine starke körperliche und geistige Belastung für den Chirurgen dar, deren Ausmaß von der Art und Dauer des Eingriffs, äußeren Umständen und der Rolle des Chirurgen abhängt.

Material und Methoden. Die Studie umfasste 101 unterschiedlich schwierige Operationen, die als erster Operateur durchgeführt oder als Assistent begleitet wurden. Mithilfe eines Trainingscomputers, einer Personenwaage und eines Thermometers wurden die OPDauer, die Herzfrequenz, der Verlust an Körperflüssigkeit, die Umgebungstemperatur und der Kalorienverbrauch registriert und ausgewertet.
Ergebnisse. Die durchschnittliche und maximale Herzfrequenz als Operateur (124 spm) waren signifikant höher als beim Assistieren (99 spm). Die subjektive psychische Belastung in den unterschiedlichen Schwierigkeitsgraden resultierte in einem Anstieg stressrelevanter Kreislaufparameter. Der durchschnittliche Gewichtsverlust während der OP betrug 0,82 kg Körpergewicht $(0-2,3 \mathrm{~kg})$. Der durchschnittliche Gewichtsverlust pro Stunde bei einer durchschnittlichen Raumtemperatur von $20,4{ }^{\circ} \mathrm{C}$ betrug $1,12 \%$ beim Operateur und 0,59\% beim Assistenten. Diskussion. Mit steigender Komplexität und OP-Dauer tritt beim Operateur eine größere physische und psychische Belastung als beim Assistenten auf. Die während und nach der
OP registrierten Kreislaufreaktionen sind vergleichbar mit denen durch Sport. Während mehrstündiger Eingriffe kommt es zu einer mäßigen Dehydratation, bei der laut Literatur mit einer negativen Beeinflussung kognitiver und motorischer Fähigkeiten gerechnet werden muss. Die Ergebnisse legen nahe, dass ein Ausgleich des Flüssigkeitsverlusts und die Anwendung von Entspannungstechniken während einer OP dazu beitragen können, mögliche Risiken durch eine herabgesetzte Leistungsfähigkeit zu minimieren.

\section{Schlüsselwörter}

Chirurgie · Dehydratation · Physischer Stress . Psychischer Stress · Pulsfrequenz

\section{Intraoperative stress in orthopaedic spine surgery. Attending surgeon versus resident}

\section{Abstract}

Introduction. Requirements for orthopaedic spine surgeons include occupational skills, concentration, physical fitness and psychological stress resistance, depending on the attending surgeon's or the resident's position.

Material and methods. This study measured and evaluated stress-relevant cardiovascular parameters during 101 spinal surgical procedures of a 40 -year old fellowship-trained spine surgeon with 12 years of practice. A training computer, personal scales and a thermometer were used to record the duration of surgery, heart rate, weight loss and calorie burning.
Results. The average maximum heart rate as an attending surgeon (124 bpm) was significantly higher than the resident's heart rate (99 bmp). A higher stress level resulted in an increasingly higher average maximum heart rate according to the duration of surgery. The mean loss of body fluids at an average room temperature of $20.4 \mathrm{C}$ after surgery was $0.82 \mathrm{~kg}$ (0 to $2.3 \mathrm{~kg}$ ). The mean loss of body weight was calculated as $1.12 \%$ of the attending surgeon versus $0.59 \%$ of the resident.

Discussion. Increasing complexity, longer duration and a higher potential of intraoperative complications arouse a strong response from the attending surgeon. The observed cardiovascular parameters are similar to those of a moderate to intense workout such as cycling. Long lasting surgeries result in a weight loss equivalent to a mild dehydration ranging from 2 to $5 \%$ of body fluids. Increasing dehydration will eventually worsen cognitive, visual and motor skills. Results of this study suggest early rehydration and utilization of mental relaxation techniques to minimize risks during prolonged, complex spine surgeries.

\section{Keywords}

Dehydration - Physiological stress . Psychological stress · Pulse rate · Surgery te durchschnittliche Gewichtsverlust pro Stunde zeigte statistisch signifikante Unterschiede. Er betrug für den Operateur $1,12 \%( \pm 0,71 \%)$ und für den Assistenten $0,59 \%( \pm 0,52 \%)(p \leq 0,05$, MannWhitney-U-Test).

Die Saaltemperatur wurde während 94 Operationen (fehlende $n=7$ ) gemessen. Trotz der klimatisierten Operationssäle lag die Spannweite der Temperaturen im Bereich von $19-24,5^{\circ} \mathrm{C}$. Der Mittelwert lag zu Beginn bei $20,4^{\circ} \mathrm{C}$ und gegen Ende der Operation bei $21,9^{\circ} \mathrm{C}$.

\section{Psychischer Stress}

Hinsichtlich der unterschiedlichen psychischen Belastung während einer Operation wurden die Eingriffe in je eine der folgenden 3 Kategorien eingeteilt: leicht, mittel und schwer. Leichte Operationen beinhalteten Routineeingriffe ohne Instrumentarium (z. B. mikroskopisch assistierte Bandscheibenoperationen, dorsale Dekompression einer Segmentetage). Zu den mittleren Operationen zählen z. B. kurzstreckige, instrumentierte Routineeingriffe wie dorsale Spondylodesen oder die transforaminelle interkor- porelle lumbale Fusion. Zu den schweren Operationen gehören z. B. technisch anspruchsvolle Revisionseingriffe, langstreckige Korrekturspondylodesen, Pedikelsubtraktionsosteotomien, einzeitige ventrodorsale Operationen und Eingriffe mit intraoperativen Komplikationen.

Als repräsentativer Kreislaufparameter für psychischen Stress wurde wiederum die Herzfrequenz bestimmt. Es wurden 43 Operationen als leicht, 19 als mittel und 29 als schwer bewertet $(n=$ 10 fehlende). Die OP-Dauer korrelierte $(r=0,53)$ mit dem subjektiven Schwierigkeitsgrad der Operation. Leichte Opera- 
Tab. 2 Unterschiedliche Schwierigkeitsgrade (psychischer Stress) der Operationen und Kreis-

laufparameter

\begin{tabular}{|llll}
\hline & Leicht & Mittel & Schwer \\
\hline Anzahl & 48 & 20 & 33 \\
\hline Durchschnittliche Herzfrequenz $(\mathrm{spm})$ & 84,4 & 98,5 & $98,8^{\mathrm{a}}$ \\
\hline Maximale Herzfrequenz $(\mathrm{spm})$ & 108,6 & 129,3 & $130,3^{\mathrm{a}}$ \\
\hline Durchschnittliche Dauer $(\mathrm{h})$ & $1: 52$ & $3: 02$ & $3: 52^{\mathrm{a}}$ \\
\hline Durschnittlicher Energieverbrauch $(\mathrm{kcal})$ & 326,5 & 794,6 & $1.013,93^{\mathrm{a}}$ \\
\hline${ }^{\text {}}$ statistisch signifikante Gruppenunterschiede $(p<0,05)$ leicht vs. schwer &
\end{tabular}

tionen dauerten durchschnittlich 1:51 h, mittlere 2:57 h und schwere 3:56 h.

Die durchschnittliche Herzfrequenz ist bei den Operationen mit hohem Schwierigkeitsgrad signifikant höher als bei den als leicht bewerteten ( $p \leq 0,05$, Kruskal-Wallis-Test). Der Unterschied zwischen den als mittel und schwer bewerteten Operationen war statistisch nicht signifikant $(p>0,05)$.

Ähnlich verhält es sich mit der maximalen Herzfrequenz. Diese ist in den höheren Schwierigkeitsgraden signifikant höher als im niedrigen $(p \leq 0,05$, Kruskal-Wallis-Test). Zwischen den als mittel und schwer bewerteten Operationen besteht auch hier kein signifikanter Unterschied $(p>0,05)$. Der Maximalwert betrug $173 \mathrm{spm}$ und der Minimalwert 85 spm (•Tab. 2).

\section{Vergleich von operativer Belastung und sportlicher Aktivität}

Um einen Maßstab für die während der Operationen auftretenden Belastungen zu erhalten, wurden Vergleichsmessungen bei unterschiedlich anstrengenden sportlichen Aktivitäten durchgeführt: Spazierengehen in der Ebene für $30 \mathrm{~min}$ (leichte Belastung), Radfahren auf dem Fahrradergometer bei $21 \mathrm{~km} / \mathrm{h}$ für $45 \mathrm{~min}$ (mittlere Belastung) und ein Zirkeltraining für 90 min (schwere Belastung).

Beim Spazierengehen wurde eine durchschnittliche Herzfrequenz von 104 spm und eine maximale Herzfrequenz von 118 spm gemessen. Während des Radfahrens konnte eine durchschnittliche Herzfrequenz von 126 spm und eine maximale Herzfrequenz von 136 spm aufgezeichnet werden. Für das Zirkeltraining ergab die Auswertung eine durchschnittliche Herzfrequenz von
128 spm und eine maximale Herzfrequenz von $178 \mathrm{spm}$.

\section{Diskussion}

\section{Körperliche Belastung}

Langes Stehen auf einer Stelle, der Umgang mit OP-Instrumenten und komplexe Operationsschritte fordern ein hohes $\mathrm{Maß}$ an Konzentration und stellen eine körperliche Belastung für den Chirurgen dar.

Aus der Literatur ist bekannt, dass vermehrter Stress zu einer Einschränkung der Leistungsfähigkeit führen kann [2]. Im schlimmsten Fall kommt es zu Fehlern, die negative Auswirkungen auf das Operationsergebnis oder gar das Leben des Patienten haben. Weiter bekannt ist, dass vermehrter Stress zu einem erhöhten Risiko für Herz-Kreislauf-Erkrankungen führt [12].

Auf Basis einer systematischen Analyse von Kreislaufparametern während 101 Operationen konnte nachgewiesen werden, dass die körperliche und psychische, d. h. mentale, Belastung für den Chirurgen in der aktiven Rolle des Operateurs signifikant höher ist als in der eher passiven Rolle des Assistenten. Dies konnte unter Berücksichtigung der Einflüsse unterschiedlicher Raumtemperaturen, der OP-Dauer und des Schwierigkeitsgrads des Eingriffs erstmals nachgewiesen und quantifiziert werden.

Das chirurgische Erfahrung nicht zwangsläufig mit einem reduzierten Stresslevel zusammenhängt, zeigten Kuhn et al. [7], die eine ähnliche Untersuchung während Koronararterienbypassoperationen durchgeführt haben. Sie kamen zu dem Ergebnis, dass die Anleitung eines weniger erfahrenen Assistenten bei Koronararterienbypassoperationen auch beim erfahrenen Chirurgen, wenn er den Eingriff anleitet und somit aktiv assistiert, einen erhöhten psychischen Stress verursachen kann.

Im Vergleich zu anderen körperlichen Aktivitäten ist die physische Belastung im Hinblick auf die durchschnittliche Herzfrequenz während wirbelsäulenchirurgischer Eingriffe aller Schwierigkeitsgrade eher gering anzusehen und kann mit dem normalen Gehen in der Ebene $(6 \mathrm{~km} / \mathrm{h})$ verglichen werden. Bei der eigenen Messung während des Spaziergangs wurde eine durchschnittliche Herzfrequenz von 104 spm erreicht. Somit lag die des Probanden der eigenen Untersuchung mit durchschnittlich 92 spm sogar noch darunter. Ein Blick auf die maximal gemessene Herzfrequenz bei 23 Operationen mit hohem Schwierigkeitsgrad zeigt jedoch, dass Spitzenwerte mit über 173 spm erreicht werden können. Diese Kreislaufparameter findet man sonst bei stark belastenden sportlichen Aktivitäten, z. B. einem intensiven Zirkeltraining $\left(\mathrm{CrossFit}^{\circledR}\right)$.

Die eigenen Ergebnisse decken sich mit denen von Bergovec et al. [3], welche den körperlichen Stress eines Orthopäden während der Implantation von Hüfttotalendoprothesen untersucht haben. Sie konnten zeigen, dass die Reaktion des kardiovaskulären Systems nicht höher ist als bei moderaten körperlichen Aktivitäten. Bei zwei der eigenen Operationen waren jedoch Höchstwerte von über $170 \mathrm{spm}$ zu verzeichnen, die sich mit der maximalen Belastung bei einem Zirkeltraining decken.

Welche Faktoren können dazu beitragen, den Stress für den Chirurgen zu reduzieren?

Dass ein moderner Operationssaal zu einer Reduktion des Stresses beitragen kann, zeigte Klein im Jahr 2010 [6], indem er die Herzfrequenz von zehn Chirurgen bei laparoskopischen Eingriffen - sowohl in modernen als auch in herkömmlichen Operationssälen - miteinander verglich. Moderne Operationssäle zeichnen sich dadurch aus, dass sie nach neuesten ergonomischen und technischen Standards konstruiert worden sind.

Ebenso kann das Erlernen und Umsetzen von Coping-Strategien zu einem besseren Umgang mit der Belastung beitra- 
gen [15]. Coping-Strategien beinhalten mentale Praktiken und auch Atemtechniken, um in heiklen Situationen Konzentration und Ruhe zu bewahren.

\section{Dehydratation}

Während wirbelsäulenchirurgischer Eingriffe sind Trinkpausen oder Ruhephasen in der Regel nicht vorgesehen bzw. nur bedingt umzusetzen, da sie den schnellen Ablauf einer Operation unterbrechen und die OP-Zeit verlängern. Während der meisten orthopädisch-unfallchirurgischen Eingriffe, so auch in der Wirbelsäulenchirurgie, müssen intraoperative Röntgenaufnahmen angefertigt werden, die das Tragen von mehreren Kilogramm schweren Bleischürzen notwendig machen. Bleischürzen sind nicht atmungsaktiv und erhöhen die Körpertemperatur während physisch anstrengender Arbeit, d.h. sie stellen eine zusätzliche thermische Belastung dar und führen zu vermehrter Transpiration und Dehydratation.

Aus der täglichen Praxis und der eigenen Untersuchung ist bekannt, dass Operateur und Assistent oft mehrere Stunden keine Möglichkeit haben, diesen Flüssigkeitsverlust auszugleichen. Zahlreiche Studien zeigen, dass mit zunehmender Dehydratation grundlegende kognitive Fähigkeiten wie das Kurzzeitgedächtnis und visuell motorische Fähigkeiten abnehmen $[1,5,13$, 14]. Nach Adan wird eine milde Dehydratation als ein Verlust von 1-2\% des Körperwassers und eine moderate Dehydratation als ein Verlust von 2-5\% des Körperwassers definiert [1]. Als kritische Grenze gilt in den vorliegenden Studien ein Verlust von $2 \%$ des Körperwassers. Gopinathan et al. [5] wiesen darauf hin, dass die Leistungsfähigkeit bei Wasserentzug und der Durchführung von körperlichen Übungen unter Hitze proportional zum Grad der Dehydratation abnimmt und ab einem Verlust von $2 \%$ des Körpergewichtes signifikant wird.

Demnach stellt sich die Frage, wie hoch ist und darf ein Flüssigkeitsverlust im Laufe einer Operation sein, bevor die Konzentrationsfähigkeit und die körperliche Leistungsfähigkeit abnehmen? Die- se Fragen lassen sich mit den eigenen Ergebnissen abschließend nicht beantworten, da sie von einer Vielzahl anderer situationsbedingter und interindividueller Faktoren abhängen.

Mit der vorliegenden Arbeit konnte gezeigt werden, dass bei wirbelsäulenchirurgischen Eingriffen nach bereits 4:40 h OP-Zeit Maximalwerte von 2,68 \% Verlust des Körpergewichts bzw. von 2,3 kg Verlust von Körperwasser erreicht werden können. Bei insgesamt acht von 101 Messungen wurden Werte über $2 \%$ Verlust des Körpergewichts festgestellt. Weitere 38 Messungen lagen im Bereich zwischen 1 und $2 \%$ und entsprachen damit einer milden Dehydratation.

In Kenntnis der aktuellen Literatur [1, $5,13,14]$ muss davon ausgegangen werden, dass in diesen acht Fällen mit moderater Dehydratation der Chirurg am Ende der Operation mit deutlichen Einschränkungen seiner körperlichen und geistigen Fähigkeiten zu rechnen hat. Wir sehen diese Tatsache darin bestätigt, dass es nach anspruchsvollen, mehrstündigen Operationen mit hohen Flüssigkeitsverlusten oft zu Kopfschmerzen, einem ausgeprägten Durstgefühl und körperlicher Erschöpfung kommen kann. Nach Meinung der Autoren können gezielte Unterbrechungen während langer, anspruchsvoller Operationen mit kurzen Trinkpausen zur Verbesserung bzw. Erhaltung einer uneingeschränkten Leistungsfähigkeit eines Chirurgen während der Operation beitragen.

\section{Psychischer Stress}

Nicht jede Operation verläuft nach Plan. Unvorhergesehene Komplikationen können zusätzlichen psychischen Stress verursachen. Psychischer Stress während einer Operation ist schwer messbar. Wir haben das subjektiv wahrgenommene Stresslevel während einer Operation in drei Kategorien eingeteilt und mit der gemessenen Herzfrequenz verglichen.

Mit der OP-Dauer steigt in der Regel auch der Schwierigkeitsgrad. Leichte Operationen dauerten durchschnittlich 1:51 h, mittlere 2:57 $\mathrm{h}$ und schwere 3:56 h. Schwere Operationen beinhalten komplexere Operationsschritte, die er- fahrungsgemäß mit einem größeren Zeitaufwand verbunden sind.

Für die statistische Auswertung wurden die als mittel und schwer bewerteten Operationen mit den leichten Operationen verglichen. Für die maximale und durchschnittliche Herzfrequenz beider Gruppen (leicht vs. schwer) zeigten sich statistisch signifikante Unterschiede $(p<0,05$, - Tab. 2). Die subjektiv als schwer empfundenen Operationen bedeuteten auch tatsächlich eine größere Belastung.

\section{Limitationen und Ausblick}

Die Kreislaufparameter dieser Studie wurden an einer Einzelperson gemessen und sind somit nicht allgemeingültig oder repräsentativ. Der Proband war männlich, körperlich gesund, mittleren Alters und hatte eine Berufserfahrung von 12 Jahren zum Zeitpunkt der Datenerhebung. Aus diesen Gründen sind ähnliche Ergebnisse bei dem überwiegenden Großteil der heute operativ tätigen Unfallchirurgen und Orthopäden in einem vergleichbaren Alter und körperlicher Konstitution zu erwarten.

Da das subjektive Stressempfinden stark von der persönlichen Erfahrung, also von der Anzahl bestimmter durchgeführter Operationen, und nicht zuletzt dem persönlichen Charakter einer Person abhängt, lassen sich dafür sicherlich keine allgemeingültigen Schlussfolgerungen ableiten. Das Stressempfinden wird also von Person zu Person starken individuellen Unterschieden unterliegen. Vielmehr ging es bei dieser Untersuchung darum nachzuweisen, dass es neben körperlichen Einflussfaktoren auch nachweislich psychische Einflussfaktoren während wirbelsäulenchirurgischer Eingriffe gibt, die mithilfe einfacher Kreislaufparameter nachweisbar und messbar sind.

Durch 101 über einen Zeitraum von mehr als einem Jahr erfasste Operationen konnten tageszeitliche und andere nicht operationsbedingte psychische Einflussfaktoren weitgehend ausgeglichen werden, sodass diese Eingriffe ein repräsentatives Bild für die Arbeitsbelastung mit typischen Eingriffen eines Wirbelsäulenchirurgen darstel- 
len. Dennoch sollten künftige Studien ein größeres Probandenkollektiv unterschiedlicher Alters- und Erfahrungsstufen beinhalten und eine größere Anzahl von Eingriffen erfasst werden, um weiterreichende Schlussfolgerungen und dezidierte Handlungsempfehlungen zur Fehlervermeidung und Optimierung der Performance von Chirurgen im OP-Saal zu ermöglichen. Auch die Erhebung weiterer kreislaufrelevanter Parameter, wie die Aufzeichnung eines Elektrokardiogramms, Messungen des Laktat- und Speichelkortisolwerts sowie Messungen der Atemfrequenz und des $\mathrm{O}_{2}$-Verbrauchs, können dazu beitragen, genauere Ergebnisse zu erzielen. Ein weiterer Kritikpunkt ist, dass die Art der Operationen (schwer, mittel, leicht) nicht unter standardisierten Bedingungen durchgeführt wurden, jedoch aufgrund der Natur der Sache auch künftig nur bedingt standardisierbar sein wird. Die Einteilung der Schwierigkeitsgrade bleibt subjektiv und wird sich von Operateur zu Operateur unterscheiden. Ein möglicher Weg zur Verbesserung wäre die Definition von Operationsschritten und Kriterien, die für den überwiegenden Anteil von Chirurgen als kritisch beurteilt werden und mit höherem psychischen Stress verbunden sind, um möglichst objektive Ergebnisse zu erzielen.

\section{Fazit für die Praxis}

Orthopädisch-wirbelsäulenchir-
urgische Eingriffe stellen für den
Operateur eine größere Belastung als
für den Assistenten dar.
- Komplexe Operationen dauern län-
ger und gehen mit einer größeren
körperlichen und psychischen Belas-
tung einher.
Größere intraoperative Flüssigkeits-
verluste während langer Operatio-
nen sollten ausgeglichen werden,
um negative Konsequenzen einer
Dehydratation zu minimieren.

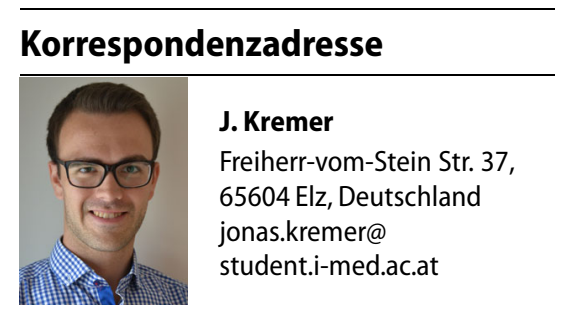

Open access funding provided by University of Innsbruck and Medical University of Innsbruck

\section{Einhaltung ethischer Richtlinien}

Interessenkonflikt. J. Kremer und M. Reinhold geben an, dass kein Interessenkonflikt besteht.

Für die vorliegende Studie war das Votum einer Ethikkommission nicht notwendig.

Open Access. Dieser Artikel wird unter der Creative Commons Namensnennung 4.0 International Lizenz (http://creativecommons.org/licenses/by/4.0/deed. de) veröffentlicht, welche die Nutzung, Vervielfältigung, Bearbeitung, Verbreitung und Wiedergabe in jeglichem Medium und Format erlaubt, sofern Sie den/die ursprünglichen Autor(en) und die Quelle ordnungsgemäßnennen, einen Linkzur Creative Commons Lizenz beifügen und angeben, ob Änderungen vorgenommen wurden.

\section{Literatur}

1. Adan A (2012) Cognitive performance and dehydration. J Am Coll Nutr 31:71-78. doi:10.1080/07315724.2012.10720011

2. Arora S, Sevdalis N, Nestel D et al (2010) The impact of stress on surgical performance: A systematic review of the literature. Surgery 147:318-330. doi:10.1016/j.surg.2009.10.007

3. Bergovec M, Orlic D (2008) Orthopaedic surgeons' cardiovascular response during total hip arthroplasty. Clin Orthop Relat Res 466:411-416. doi:10.1007/s11999-007-0037-y

4. Enerson DM, Eisenfeld LI, Kajikuri H (1967) Heat and moisture trapping beneath surgical face masks: a consideration of factors affecting the surgeon's discomfort and performance. Surgery 62:1007-1016

5. Gopinathan PM, Pichan G, Sharma VM (1988) Role of dehydration in heat stress-induced variations in mental performance. Arch Environ Health 43:15-17. doi:10.1080/00039896.1988.9934367

6. Klein M, Andersen L, Alamili M (2010) Psychological and physical stress in surgeons operating in a standard or modern operating room. Surg Laparosc Endosc Percutan Tech 20(4):237-242

7. Kuhn EW, Choi Y-H, Schönherr M et al (2013) Intraoperative stress in cardiac surgery: attendings versus residents. J Surg Res 182:e43-49. doi:10.1016/j.jss.2012.11.011

8. LiY, Tokura H, Guo YP et al (2005) Effects of wearing N95 and surgical facemasks on heart rate, thermal stress and subjective sensations. Int Arch Occup Environ Health 78:501-509. doi:10.1007/s00420 004-0584-4
9. Müller M (2014) Risikomanagement und Sicherheitsstrategien der Luftfahrt - ein Vorbild für die Medizin?ZAllgemeinmed 79:339-344

10. Nielsen R, Gwosdow AR, Berglund LG, DuBois AB (1987) The effect of temperature and humidity levels in a protective mask on user acceptability during exercise. Am Ind Hyg Assoc J 48:639-645. doi:10.1080/15298668791385336

11. Roberge R, Kim JH, Coca A (2012) Protective facemask impact on human thermoregulation: an overview. Ann Occup Hyg 56:102-112. doi:10.1093/annhyg/mer069

12. Sloan RP, Shapiro PA, Bagiella E et al (1994) Effect of mental stress throughout the day on cardiac autonomic control. Biol Psychol 37:89-99

13. Szinnai G, Schachinger H, Arnaud MJ et al (2005) Effect of water deprivation on cognitive-motor performance in healthy men and women. Am J Physiol Regul Integr Comp Physiol 289:R275-280. doi:10.1152/ajpregu.00501.2004

14. Tomporowski PD, Beasman K, Ganio MS, Cureton K (2007) Effects of dehydration and fluid ingestion on cognition. Int J Sports Med 28:891-896. doi:10.1055/s-2007-965004

15. Wetzel CM, Kneebone RL, Woloshynowych $M$ et al (2006) The effects of stress on surgical performance. Am J Surg 191:5-10. doi:10.1016/j.amjsurg.2005.08.034

16. http://dr-mach.de. Zugegriffen:23. Oktober 2015

17. http://aok-bv.de. Zugegriffen: 10. Oktober 2015 